\title{
Assessment of 3-month changes in bone microstructure under anti-TNFa therapy in patients with rheumatoid arthritis using high-resolution peripheral quantitative computed tomography (HR-pQCT)
}

\author{
Tomohiro Shimizu ${ }^{1,2^{*}+}$, Hyo Jin Choi ${ }^{1,3+}$, Ursula Heilmeier ${ }^{1}$, Matthew Tanaka ${ }^{1}$, Andrew J. Burghardt ${ }^{1}$,
} Jingshan Gong ${ }^{1}$, Nattagan Chanchek', Thomas M. Link', Jonathan Graf', John B. Imboden ${ }^{4}$ and Xiaojuan Li ${ }^{1}$

\begin{abstract}
Background: Although one study showed minimal progression of erosions in patients with rheumatoid arthritis (RA) one year after TNFa inhibition therapy, no studies have investigated very early bone changes after initiation of anti-TNFa treatment. We investigated the effects of 3-month anti-TNFa treatment on bone erosion progression and bone microarchitecture in RA patients using high-resolution peripheral quantitative computed tomography (HR-pQCT).

Methods: Patients with RA ( $n=27$ ) (17 in the anti-TNFa and 10 in the MTX-only group) underwent assessment of disease activity score in 28 joints (DAS-28), radiographs, 3-T magnetic resonance imaging (MRI) and HR-pQCT of metacarpophalangeal and wrist joints at baseline and 3 months. HR-pQCT-derived erosion volume, joint volume/ width and bone microarchitecture were computed and joint destruction was assessed using Sharp and RAMRIS scorings on radiographs and MRI, respectively.

Results: Overall, 73 erosions were identified by HR-pQCT at baseline. Over 3 months, the anti-TNFa group had decreased mean erosion volume; increased erosion volume was observed in one clinical non-responder. The MTX-only group in contrast, trended toward increasing erosion volume despite low disease activity. In the antiTNFa group, joint-space width and volume of MCP joints decreased significantly and was positively correlated with erosion volume changes $\left(R^{2}=0.311, p=0.013 ; R^{2}=0.527, p=0.003\right.$, respectively). In addition, erosion volume changes were significantly negatively correlated with changes in trabecular bone mineral density $\left(R^{2}=0.353, p=0.020\right)$ in this group. We observed significant correlation between percentage change in erosion volume and change in DAS-28 erythrocyte sedimentation rate and C-reactive protein CRP scores $\left(R^{2}=0.558, p<0.001 ; R^{2}=0.745, p<0.001\right.$, respectively) in all patients.

(Continued on next page)
\end{abstract}

\footnotetext{
* Correspondence: simitom@wg8.so-net.ne.jp

${ }^{\dagger}$ Equal contributors

'Department of Radiology \& Biomedical Imaging, Musculoskeletal

Quantitative Imaging Research, University of California San Francisco, 185 Berry Street, Suite 350, San Francisco, CA 94107, USA

2Department of Orthopedic Surgery, Graduate School of Medicine, Hokkaido University, Sapporo, Japan

Full list of author information is available at the end of the article
}

\section{) Biomed Central}

(c) The Author(s). 2017 Open Access This article is distributed under the terms of the Creative Commons Attribution 4.0 International License (http://creativecommons.org/licenses/by/4.0/), which permits unrestricted use, distribution, and reproduction in any medium, provided you give appropriate credit to the original author(s) and the source, provide a link to the Creative Commons license, and indicate if changes were made. The Creative Commons Public Domain Dedication waiver (http://creativecommons.org/publicdomain/zero/1.0/) applies to the data made available in this article, unless otherwise stated. 
(Continued from previous page)

Conclusions: Using HR-pQCT, our data suggest that anti-TNFa treatment prevents erosion progression and deterioration of bone microarchitecture within the first 3 months of treatment, one patient not responding to treatment, had significant progression of bone erosions within this short time period. Patients with low disease activity scores (<3.2) can have continuous HR-pQCT-detectable progression of erosive disease with MTX treatment only. HR-pQCT can be a sensitive, powerful tool to quantify bone changes and monitor RA treatment short term (such as 3 months).

Keywords: Rheumatoid arthritis, Anti-tumor necrosis factor alpha, High-resolution peripheral quantitative computed tomography, Bone erosion

\section{Background}

Rheumatoid arthritis (RA) is progressive chronic inflammatory arthritis characterized by bone and cartilage erosions and joint damage [1]. Bone erosions are usually irreversible and may occur in the first few months of RA onset [2]. The onset of bone erosions reflects a more severe course of RA, and is associated with a poor quality of life and increased mortality [3].

Tumor necrosis factor alpha (TNF $\alpha)$ is a proinflammatory cytokine involved in the pathogenesis of RA. It induces the production and release of other proinflammatory cytokines (IL-1, IL-6, IL-8), and also stimulates osteoclast activation in bone, eventually leading to bone erosions [3]. Several inhibitors of TNF $\alpha$ are currently available on the market and have proven to be a useful strategy to suppress inflammation in patients with RA. Thus, anti-TNF $\alpha$ treatments have been shown to prevent erosion progression compared to a treatment with methotrexate (MTX) alone [4-7]. In those studies, either the radiography-based modified Sharp scores [4-7] or magnetic resonance imaging (MRI) was used to determine erosion progression $[8,9]$.

High-resolution peripheral quantitative computed tomography (HR-pQCT), is a very sensitive imaging tool to detect bone erosions [10] and to evaluate cortical and trabecular bone mineral density (BMD) and microstructure in RA [11]. It has also been applied in studies of psoriatic arthritis [12] and hand osteoarthritis [13]. As firstgeneration HR-pQCT scanners allow for very high image resolution with an isotropic voxel size of $80 \mu \mathrm{m}$, HRpQCT has also evolved as a very valuable imaging tool to detect bone erosions and to evaluate cortical and trabecular BMD and microstructure. Using conventional multidetector computed tomography (CT) with a voxel size to $400 \mu \mathrm{m} \times 400 \mu \mathrm{m} \times 400 \mu \mathrm{m}$, Moller-Dohn et al. [14] identified minimal erosive progression one year after TNF $\alpha$ inhibition therapy in patients with RA. However, no studies so far have used HR-pQCT to examine the very early changes in bone microarchitecture (erosion and bone density and structures) after anti-TNF $\alpha$ treatment.

Thus, the goal of our study was to employ HR-pQCT to characterize the bone microstructural and erosive changes in patients with low disease activity on MTX over 3 months and in patients with high disease activity 3 months after initiation of anti-TNF $\alpha$ therapy. We hypothesized that anti-TNF $\alpha$ treatment would decrease the progression of bone erosions in RA as detectable using HR-pQCT.

\section{Methods \\ Subjects}

We prospectively enrolled patients in our RA cohort from March 2014 to October 2016. All patients were 18 years of age or older and fulfilled the 2010 American College of Rheumatology/European League Against Rheumatism (EULAR) classification criteria for RA [15]. Concurrent use of prednisone was permitted in doses $\leq 10 \mathrm{mg} /$ day. Two groups of patients were recruited. Inclusion criteria for patients in the methotrexate (MTX) only treatment group (MTX-only group) were low disease activity with a disease activity score in 28 joints (DAS-28) $\leq 3.2$ [16] during the last 2 months prior to the baseline visit; MTX at a stable dose for $\geq 8$ weeks; no biologic therapy during the previous 6 months; and no anticipated biologic therapy for the next year. Inclusion criteria for patients in the combined MTX and anti-TNF $\alpha$ treatment group (anti-TNF $\alpha$ group) were moderate to severe RA with DAS-28 > 3.2; these patients were scheduled to initiate anti-TNFa (certolizumab) in addition to an ongoing MTX regimen. DAS scores were used to assess clinical response from baseline to 3 months. HR-pQCT was performed at baseline (prior to anti-TNF $\alpha$ initiation in the anti-TNF $\alpha$ group) and after 3 months. DAS scores were obtained by board-certified rheumatologists (JBI and JG) on the same day of imaging. Serum samples were collected to measure C-reactive protein (CRP) and erythrocyte sedimentation rate (ESR) at baseline and 3-month follow up. The Institutional Review Board (IRB) for Human Research approved this study design (IRB\# 12-10418) and written informed consent was obtained from all subjects prior to participating in the study. 


\section{Imaging studies \\ HR-pQCT}

All subjects were imaged with a first-generation HRpQCT system (XtremeCT, Scanco Medical AG, Bruttisellen, Switzerland). HR-pQCT imaging was performed at the metacarpophalangeal (MCP) and wrist joints and distal radius of the dominant hand [17]. The distal radius scan was carried out in a manufacturerprovided forearm cast using the default imaging protocol [18], which covered $9.02 \mathrm{~mm}$ (110 slices) starting $9.5 \mathrm{~mm}$ distal to the mid-point of the radiocarpal joint surface of the radial head (Fig. 1). For the MCP and wrist joint acquisition, the patient's forearm was immobilized in a palm-down orientation inside a custom carbon-fiber holder fit with an ergonomic thermoplastic molding. The MCP scan covered $18.04 \mathrm{~mm}$ (220 slices). The first slice was located $2 \mathrm{~mm}$ distal to the reference line position. The reference line was placed at the distal margin of the phalangeal base of the distal-most joint (MCP2 or MCP3). The total scan time for the MCP joints was approximately 6 minutes, with an effective radiation dose of $8.4 \mu \mathrm{Sv}$. The wrist scan covered $27.06 \mathrm{~mm}$ (330 slices). The first slice was located $2 \mathrm{~mm}$ distal to the distal margin of the radio-scaphoid joint, intersecting the lateral edge of the joint surface of the scaphoid (Fig. 1). The total scan time for the wrist was approximately $9 \mathrm{~min}$, while the effective dose was $12.6 \mu \mathrm{Sv}$. All scans were monitored for motion artifacts, and the scan was repeated once if the image grade exceeded 2 on the standard 5-point image quality scale [19].

\section{HR-pQCT image analysis}

All HR-pQCT images were analyzed by a rheumatologist (TS, HJC). Intra-reader and inter-reader reproducibility was assessed in both MCP joints and the distal radius of 14 patients. We calculated intraobserver reproducibility in the bone erosion analysis on the basis of five consecutive measurements, and calculated interobserver reliability from the measurements by three independent readers (two board-certified rheumatologists (TS, HJC) and a board-certified radiologist (JSG)). Radiographs were analyzed jointly by a musculoskeletal radiologist (TML) and two rheumatologists (TS and $\mathrm{HJC}$ ), and MRI images were analyzed jointly by two radiologists (NC, TML). Standard HR-pQCT bone measurements at the distal radius, including BMD, cortical or trabecular bone microarchitecture were calculated semi-automatically (TS, MT) [20]. Analogous bone microarchitectural parameters were calculated in the distal head of the second and third metacarpal ( $\mathrm{MCH} 2$ and $\mathrm{MCH} 3)$. The analyzed region of interest was scaled in the slice-wise direction to span the distal $12 \%$ of the full metacarpal length as validated in a previous study [21]. The length of each metacarpal was measured (TS, HJC) on the baseline radiographs of the ipsilateral hand and used to normalize BMD and cortical thickness data in each bone. Three-dimensional measures of mean joint-space width (JSW) and volume were measured using an automated pipeline developed in our group [22]. All readers were blinded to the clinical information and there was a time interval of at least 4 weeks between the readings derived from the different imaging modalities.

Bone erosions in the MCP were evaluated from the HRpQCT images following the recommended definitions and procedures developed by the Study GrouP for XTrEmeCT in Rheumatoid Arthritis (SPECTRA) [23]. We adapted analogous definitions and procedures for evaluating erosions at the wrist. An erosion was defined as a sharply demarcated cortical break spanning two or more consecutive axial slices and confirmed in the sagittal or coronal planes. If multiple erosions were present in the same quadrant (palmar, dorsal, radial or ulnar quadrant), the biggest erosion was measured. The maximum width and depth of erosions at the second and third $\mathrm{MCH}$ and phalangeal base $(\mathrm{PB})$, and radial head were measured. Erosion volume was estimated using an ellipsoid model:

$$
\mathrm{V}_{\text {erosion }}=\frac{4}{3} \pi \times \frac{\text { width }}{2} \times \text { depth } \times \frac{\text { perpendicular width }}{2}
$$

Total erosion volume was calculated as the summation of all individual erosion volumes from $\mathrm{MCH} 2, \mathrm{MCH} 3$,
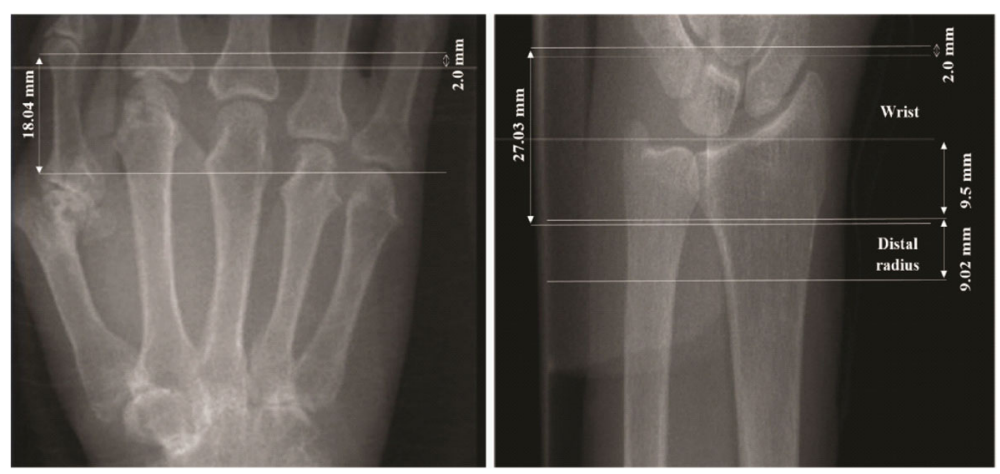

Fig. 1 Scout film of metacarpophalangeal and wrist joints and distal radius on high-resolution peripheral quantitative images 
PB2, PB3 and radial head. All erosion measurements and metacarpal lengths were performed using the open-source digital imaging and communications in medicine (DICOM) viewer Osirix V 7.0 (Nema, Rosslyn, VA, USA).

\section{Radiographs and MRI and scoring}

Bilateral dorsopalmar and oblique hand and feet radiographs were obtained at baseline. The bilateral radiographs were further evaluated using the well-known, modified Sharp/van der Heijde score for radiographs $[24,25]$.

MRI scans of the dominant wrist joints were acquired on a 3-T MR unit (MR 750 Wide Bore; GE Healthcare, Milwaukee, WI, USA) with an eight-channel phased array wrist coil (In vivo, Gainesville, FL, USA). Patients were examined in a supine position with their arm resting on the side of the body. To assess the bone marrow edema (BME) pattern, coronal T2-weighted iterative decomposition of water and fat with echo asymmetry and least-squares estimation (IDEAL) fast spin echo (FSE) water images (repetition time (TR)/echo time $(\mathrm{TE})=3500 / 50 \mathrm{~ms}$, in-plane resolution $=0.2 \mathrm{~mm}$, slice thickness $=2 \mathrm{~mm}$ ) were performed. Coronal T1weighted IDEAL spoiled gradient echo (SPGR) images $(\mathrm{TR} / \mathrm{TE}=15.3 / 2.9 \mathrm{~ms}$, in-plane resolution $=0.2 \mathrm{~mm}$, slice thickness $=1 \mathrm{~mm}$ ) served for scoring erosion and joint-space narrowing. Coronal T1-weighted IDEAL FSE images $(\mathrm{TR} / \mathrm{TE}=600 / 9.9 \mathrm{~ms}$, in-plane resolution $=$ $0.44 \mathrm{~mm}$, slice thickness $=2 \mathrm{~mm}$ ) pre and post gadolinium injection were used to assess synovitis and erosions. MRI images were evaluated using the Outcome Measures in Rheumatology (OMERACT) RA-MRI scoring (RAMRIS) system to assess erosion, BME pattern, joint-space narrowing and synovitis $[26,27]$.

\section{Statistical analysis}

The paired $t$ test was performed to compare patient characteristics and bone measurements within groups from baseline to 3 months. For intra-reader and interreader reproducibility of erosion assessment, Cohen's kappa and coefficient variance (CV \%) were calculated. Additionally, the least significant change (LSC) at $95 \%$ confidence level was calculated from CV\%; $p$ values $<0.05$ were considered statistically significant. All statistical analyses were performed using PASW Statistics ver. 18.0 (IBM Co., Armonk, NY, USA).

\section{Results}

\section{Clinical results}

Twenty-seven patients with clinically diagnosed RA were enrolled. There were 17 patients on anti-TNF $\alpha$ treatment combined with MTX treatment (anti-TNF $\alpha$ group) (6 with moderate, and 11 with high disease activity according to DAS-28-ESR at baseline), while 9 patients were on MTX treatment only (MTX-only group) (4 in remission and 5 with low disease activity). Table 1 summarizes the baseline characteristics of the two study groups. There were 9 patients in the MTX-only group and 14 in the anti-TNF $\alpha$ group who were followed up completely. Over the course of 3 months, among the patients on anti-TNF $\alpha$ treatment, six patients qualified as good responders, seven were moderate, and one was a non-responder, in accordance with the EULAR response criteria [16]. One patient could not be followed because of pregnancy and two other patients in the anti-TNFa group were lost to follow up for unknown reasons. No significant differences in demographics and baseline image measures were observed between the patients with and without follow up. In addition, over the 3month treatment period, most of the clinical disease

Table 1 Characteristics of patients with rheumatoid arthritis - combined or by group - at baseline

\begin{tabular}{llll}
\hline & All patients $(n=27)$ & MTX-only group $(n=10)$ & MTX+anti-TNFa group $(n=17)$ \\
\hline Age (years), mean \pm SD & $51.9 \pm 15.4$ & $58.6 \pm 15.8$ & $47.7 \pm 13.8$ \\
Sex (female:male) & $22: 5$ & $7: 3$ & $15: 2$ \\
Ethnicity & 25 & & 15 \\
$\quad$ Hispanic or Latino & 1 & 10 & 1 \\
$\quad$ Native Hawaiian or other Pacific Islanders & 1 & 0 & 1 \\
$\quad$ African American & $6.5 \pm 5.4$ & 0 & $5.7 \pm 5.6$ \\
Disease duration (years), mean \pm SD & $28.3 \pm 7.1$ & $7.1 \pm 5.2$ & $30.1 \pm 7.6$ \\
Body mass index (kg/m ${ }^{2}$ ), mean \pm SD & 14 & $24.5 \pm 5.5$ & 11 \\
Seropositivity of rheumatoid factor & 19 & 3 & 12 \\
$\quad$ Anti-CCP antibody & $4.5 \pm 3.8$ & 7 & $4.9 \pm 3.6$
\end{tabular}

MTX methotrexate, TNFa Tumor necrosis factor alpha, DAS-28 disease activity score in 28 joints, CCP citrullinatcd protein 
activity parameters significantly improved in the antiTNF $\alpha$ group: both DAS-28-CRP and DAS-28-ESR exhibited significant decreases in this patient group (Table 2).

\section{Bone erosion analysis}

Overall, 73 erosions were detected by HR-pQCT. In the anti-TNF $\alpha$ group, erosions were located on the MCP2 $(n=23)$, MCP3 $(n=13)$, and distal radius $(n=11)$; the distribution was 7, 4 and 15, respectively, in the MTXonly group. During the 3-month follow-up period, the number of erosions in the both groups did not change (Table 3). In the anti-TNF $\alpha$ group, mean erosion volume throughout the joints numerically decreased over the 3 months, being statistically significant at MCP3 $(p=0.014)$ in all patients, and at MCP2 and MCP3 ( $p=0.048$ and $p=0.014$, respectively), if we excluded the one non-responder. For the MTX-only group, on the contrary, we observed a slight increase overall in mean erosion volume throughout the joints, most pronounced at MCP2 $(p=0.006)$ and MCP3 $(p=0.019)$ (Table 3).

The kappa scores $(k)$ for intra-reader and interreader reproducibility for detecting erosions were $0.970(p<0.001)$ and $0.818(p=0.001)$, respectively. For the measurement of erosion volume, intra-reader agreement was $3.43 \%$ (root mean square (RMS) $\% \mathrm{CV}$ ), and inter-reader agreement was $3.92 \%$ (RMS $\% \mathrm{CV}$ ). The volume changes of 17 erosions (out of 26 erosions) in the MTX-only group and 32 erosions (out of 47 erosions) in the anti-TNF $\alpha$ group, respectively, exceeded the least significant change (LSC).

\section{Joint space analysis}

We next investigated changes in joint space geometry, namely in width and volume, from baseline to 3 months using a 3D joint space quantification derived from HRpQCT images. In the anti-TNF $\alpha$ group, we consistently observed a decrease in joint space volume and joint space width throughout the measured joint sites over the 3-month follow-up period (Table 3). The opposite pattern was observed in the MTX only group, where we observed almost no change or a trending increase in joint volume over time.

\section{Microarchitecture analysis}

Additionally, we investigated other HR-pQCT measurements in our study to assess changes in bone

Table 2 Clinical features and radiological imaging scores in each treatment group from baseline and 3 months follow up in all patients with rheumatoid arthritis who had a 3-month follow-up visit

\begin{tabular}{|c|c|c|c|c|c|c|c|c|}
\hline \multirow{2}{*}{$\begin{array}{l}\text { Total }(n=22) \\
\text { Mean } \pm \text { SD }\end{array}$} & \multicolumn{4}{|c|}{ MTX only group $(n=9)$} & \multicolumn{4}{|c|}{ MTX + anti-TNFa group $(n=13)$} \\
\hline & Baseline & 3 Months & Changes & $p$ value* & Baseline & 3 Months & Changes & $p$ value* \\
\hline \multicolumn{9}{|l|}{ Disease activity } \\
\hline Swollen joints & $0.7 \pm 1.0$ & $1.6 \pm 1.8$ & $0.9 \pm 1.8$ & 0.184 & $14.5 \pm 3.9$ & $6.3 \pm 4.8$ & $-8.2 \pm 6.6$ & $<0.001$ \\
\hline Tender joints & $0.7 \pm 1.1$ & $1.0 \pm 1.3$ & $0.3 \pm 1.2$ & 0.438 & $9.3 \pm 6.2$ & $4.4 \pm 9.5$ & $-4.9 \pm 9.9$ & 0.086 \\
\hline Global assessment of patient & $7.4 \pm 15.3$ & $16.3 \pm 25.7$ & $8.9 \pm 19.6$ & 0.210 & $62.2 \pm 16.8$ & $41.4 \pm 31.2$ & $-20.8 \pm 26.6$ & 0.015 \\
\hline Physician & $11.7 \pm 10.9$ & $14.1 \pm 12.6$ & $2.4 \pm 9.4$ & 0.459 & $42.8 \pm 10.7$ & $26.4 \pm 12.1$ & $-16.4 \pm 18.2$ & 0.005 \\
\hline ESR unm h) (mm/h) & $18.0 \pm 22.3$ & $21.0 \pm 18.3$ & $3.0 \pm 10.9$ & 0.435 & $32.2 \pm 21.9$ & $29.7 \pm 21.6$ & $-2.4 \pm 12.9$ & 0.490 \\
\hline CRP tmg/L) (mg/L) & $2.1 \pm 1.1$ & $4.6 \pm 3.5$ & $2.4 \pm 2.9$ & 0.034 & $21.9 \pm 28.1$ & $10.4 \pm 9.8$ & $-11.5 \pm 22.2$ & 0.088 \\
\hline DAS-28-ESR & $1.9 \pm 0.9$ & $2.6 \pm 0.9$ & $0.7 \pm 1.2$ & 0.106 & $5.7 \pm 1.1$ & $3.8 \pm 1.2$ & $-2.0 \pm 0.9$ & $<0.001$ \\
\hline DAS-28-CRP & $1.8 \pm 0.7$ & $2.4 \pm 0.8$ & $0.6 \pm 0.6$ & 0.032 & $5.4 \pm 0.9$ & $3.5 \pm 1.0$ & $-1.7 \pm 1.1$ & $<0.001$ \\
\hline HAQ & $0.5 \pm 0.7$ & $0.7 \pm 0.8$ & $0.2 \pm 0.3$ & 0.084 & $1.6 \pm 0.7$ & $1.0 \pm 0.7$ & $-0.6 \pm 0.6$ & 0.001 \\
\hline \multicolumn{9}{|l|}{ Modified SHARP score } \\
\hline Total & $4.7 \pm 6.0$ & NA & NA & & $26.1 \pm 42.0$ & NA & NA & \\
\hline Erosion & $1.6 \pm 2.6$ & & & & $13.5 \pm 26.8$ & & & \\
\hline JSN & $3.1 \pm 4.9$ & & & & $12.5 \pm 17.0$ & & & \\
\hline \multicolumn{9}{|l|}{ RAMRIS score } \\
\hline JSN & $0.2 \pm 0.4$ & $0.3 \pm 0.7$ & $0.1 \pm 0.3$ & 0.347 & $5.9 \pm 8.4$ & $5.9 \pm 8.5$ & $0.0 \pm 0.4$ & $<1.000$ \\
\hline Synovitis & $3.2 \pm 2.1$ & $3.1 \pm 2.1$ & $-0.1 \pm 0.9$ & 0.598 & $4.9 \pm 2.6$ & $3.9 \pm 2.3$ & $-1.0 \pm 2.1$ & 0.126 \\
\hline Bone erosion & $2.6 \pm 2.2$ & $2.6 \pm 2.6$ & $0.0 \pm 0.9$ & $<1.000$ & $20.5 \pm 33.4$ & $21.2 \pm 33.3$ & $0.6 \pm 1.2$ & 0.171 \\
\hline Bone edema & $4.9 \pm 4.0$ & $4.9 \pm 3.6$ & $0.0 \pm 1.1$ & $<1.000$ & $9.7 \pm 7.8$ & $7.1 \pm 6.3$ & $-2.6 \pm 3.8$ & 0.105 \\
\hline
\end{tabular}

MTX methotrexate, TNFa tumor necrosis factor alpha, ESR erythrocyte sedimentation rate, CRP C-reactive protein, DAS-28 disease activity score $28, H A Q$ health assessment questionnaire score, JSN joint space narrowing, NA not applicable, RAMRIS Outcome Measures in Rheumatology (OMERACT) rheumatoid arthritis-magnetic resonance imaging scoring. *Paired $t$ test 
Table 3 Comparison of erosion volume and bone parameters by high-resolution peripheral quantitative computed tomography between baseline and 3 months in each treatment group

\begin{tabular}{|c|c|c|c|c|c|c|c|c|}
\hline \multirow{2}{*}{$\begin{array}{l}\text { Total }(n=19) \\
\text { Mean } \pm \text { SD }\end{array}$} & \multicolumn{4}{|c|}{ MTX-only group $(n=9)$} & \multicolumn{4}{|c|}{ MTX + anti-TNFa group $(n=13)$} \\
\hline & Baseline & 3 Months & Changes (A) & $p$ value $^{*}$ & Baseline & 3 Months & Changes (A) & $p$ value* \\
\hline \multicolumn{9}{|l|}{$\overline{\mathrm{MCP} 2}$} \\
\hline Erosion number $(n)$ & 7 & 7 & & & 23 & 23 & & \\
\hline Erosion volume $\left(\mathrm{mm}^{3}\right)$ & $6.7 \pm 6.4$ & $7.5 \pm 6.4$ & $0.9 \pm 0.5$ & 0.006 & $41.4 \pm 65.5$ & $37.6 \pm 60.3$ & $-3.8 \pm 5.2$ & 0.048 \\
\hline \multicolumn{9}{|l|}{ Joint space } \\
\hline Width (mm) & $1.81 \pm 0.14$ & $1.80 \pm 0.13$ & $-0.01 \pm 0.07$ & 0.705 & $1.87 \pm 0.20$ & $1.80 \pm 0.15$ & $-0.07 \pm 0.09$ & 0.028 \\
\hline Volume $\left(\mathrm{mm}^{3}\right)$ & $133.6 \pm 17.3$ & $134.6 \pm 16.0$ & $1.0 \pm 5.5$ & 0.606 & $125.4 \pm 20.5$ & $118.7 \pm 17.0$ & $-6.68 \pm 7.08$ & 0.001 \\
\hline \multicolumn{9}{|c|}{$\mathrm{MCH} 2$ microarchitecture BMD (mg/cm3) } \\
\hline Total & $279.9 \pm 34.2$ & $277.4 \pm 40.2$ & $-6.2 \pm 16.1$ & 0.288 & $316.2 \pm 34.7$ & $327.8 \pm 45.9$ & $11.6 \pm 33.0$ & 0.268 \\
\hline Trabecular & $236.2 \pm 26.9$ & $233.6 \pm 33.2$ & $-2.6 \pm 20.4$ & 0.731 & $259.0 \pm 25.6$ & $266.9 \pm 41.0$ & $7.8 \pm 26.8$ & 0.353 \\
\hline \multicolumn{9}{|l|}{ MCP3 } \\
\hline Erosion number $(n)$ & 4 & 4 & & & 13 & 13 & & \\
\hline Erosion volume (mm3) & $2.3 \pm 11.5$ & $3.1 \pm 1.2$ & $0.8 \pm 0.5$ & 0.019 & $21.6 \pm 31.5$ & $18.9 \pm 29.0$ & $-2.7 \pm 2.5$ & 0.014 \\
\hline \multicolumn{9}{|l|}{ Joint space } \\
\hline Width (mm) & $1.72 \pm 0.14$ & $1.74 \pm 0.15$ & $0.02 \pm 0.05$ & 0.226 & $1.83 \pm 0.12$ & $1.74 \pm 0.14$ & $-0.10 \pm 0.07$ & 0.001 \\
\hline Volume $\left(\mathrm{mm}^{3}\right)$ & $138.9 \pm 17.0$ & $142.1 \pm 20.2$ & $3.2 \pm 4.3$ & 0.069 & $131.9 \pm 24.1$ & $125.6 \pm 21.4$ & $-4.3 \pm 4.6$ & 0.003 \\
\hline \multicolumn{9}{|c|}{$\mathrm{MCH} 3$ microarchitecture BMD $\left(\mathrm{mg} / \mathrm{cm}^{3}\right)$} \\
\hline Total & $279.9 \pm 28.0$ & $280.8 \pm 25.0$ & $1.0 \pm 7.0$ & 0.706 & $324.5 \pm 35.7$ & $321.8 \pm 34.8$ & $-2.7 \pm 8.4$ & 0.298 \\
\hline Trabecular & $238.9 \pm 20.1$ & $241.9 \pm 18.1$ & $3.0 \pm 8.1$ & 0.322 & $269.4 \pm 26.7$ & $267.6 \pm 26.1$ & $-1.7 \pm 8.5$ & 0.513 \\
\hline \multicolumn{9}{|l|}{ Distal radius } \\
\hline Erosion number $(n)$ & 15 & 15 & & & 11 & 11 & & \\
\hline Erosion volume $\left(\mathrm{mm}^{3}\right)$ & $21.1 \pm 26.4$ & $23.4 \pm 29.1$ & $1.6 \pm 3.3$ & 0.075 & $199.9 \pm 362.6$ & $188.4 \pm 331.4$ & $-15.5 \pm 31.7$ & 0.211 \\
\hline \multicolumn{9}{|l|}{ Joint space } \\
\hline Width $\left(\mathrm{mm}^{3}\right)$ & $2.04 \pm 0.24$ & $2.04 \pm 0.18$ & $-0.01 \pm 0.12$ & 0.981 & $2.09 \pm 0.29$ & $2.11 \pm 0.29$ & $0.01 \pm 0.17$ & 0.758 \\
\hline Volume $\left(\mathrm{mm}^{3}\right)$ & $249.8 \pm 29.3$ & $261.1 \pm 30.5$ & $11.3 \pm 22.3$ & 0.197 & $247.5 \pm 67.1$ & $240.2 \pm 71.2$ & $-7.3 \pm 25.0$ & 0.356 \\
\hline \multicolumn{9}{|c|}{ Wrist microarchitecture BMD $\left(\mathrm{mg} / \mathrm{cm}^{3}\right)$} \\
\hline Total & $272.3 \pm 61.3$ & $272.8 \pm 60.3$ & $0.6 \pm 4.3$ & 0.709 & $356.0 \pm 58.6$ & $354.0 \pm 58.4$ & $-2.0 \pm 7.1$ & 0.357 \\
\hline Cortical & $793.7 \pm 82.9$ & $792.1 \pm 83.2$ & $-1.6 \pm 9.8$ & 0.663 & $873.4 \pm 42.7$ & $874.9 \pm 43.7$ & $1.5 \pm 9.7$ & 0.607 \\
\hline Trabecular & $148.0 \pm 31.0$ & $148.6 \pm 30.8$ & $0.6 \pm 2.7$ & 0.543 & $174.5 \pm 53.7$ & $172.0 \pm 51.2$ & $-2.5 \pm 5.1$ & 0.115 \\
\hline Cortical thickness $(\mu \mathrm{m})$ & $607.8 \pm 200.4$ & $606.7 \pm 199.5$ & $3.8 \pm 21.2$ & 0.900 & $824.6 \pm 142.7$ & $830.8 \pm 140.8$ & $6.2 \pm 27.3$ & 0.450 \\
\hline \multicolumn{9}{|l|}{ Trabecular } \\
\hline Number & $1.82 \pm 0.14$ & $1.80 \pm 0.17$ & $-0.02 \pm 0.15$ & 0.748 & $1.97 \pm 0.45$ & $1.94 \pm 0.42$ & $-0.03 \pm 0.10$ & 0.315 \\
\hline Thickness ( $\mu \mathrm{m})$ & $68.9 \pm 12.6$ & $69.9 \pm 12.6$ & $1.4 \pm 5.0$ & 0.441 & $72.9 \pm 10.8$ & $73.1 \pm 10.2$ & $0.2 \pm 4.1$ & 0.898 \\
\hline Separation $(\mu \mathrm{m})$ & $485.7 \pm 51.1$ & $491.2 \pm 60.0$ & $5.6 \pm 45.6$ & 0.740 & $471.5 \pm 171.7$ & $475.1 \pm 156.3$ & $3.6 \pm 34.8$ & 0.725 \\
\hline
\end{tabular}

The distal radius joint space was measured at the radio-lunar joint. MTX methotrexate, TNFa tumor necrosis factor alpha, MCH metacarpal heads, $B M D$ bone mineral density. *Paired $t$ test. Bold characters mean $p<0.05$

microarchitecture at the MCP and distal radius. There were no differences in bone microarchitecture from baseline to 3 months with either of the treatments.

\section{Correlations of parameters evaluated by HR-pQCT}

To better understand the 3-month changes in bone microstructure induced by anti-TNF $\alpha$ treatment, we assessed the correlation between changes in joint space, in bone erosion and in bone microarchitecture in the
anti-TNF $\alpha$ group (Fig. 2). At the MCP joints, there was strong significant correlation between changes in joint space width and volume as expected (Fig. 2a). Changes in bone erosion volume were significantly correlated with changes in joint space width and volume (Fig. 2b). Changes in total and trabecular BMD were significantly negatively correlated with changes in erosion volume, joint space width and volume (Fig. 2c). The other parameters did not demonstrate significant correlation. In 


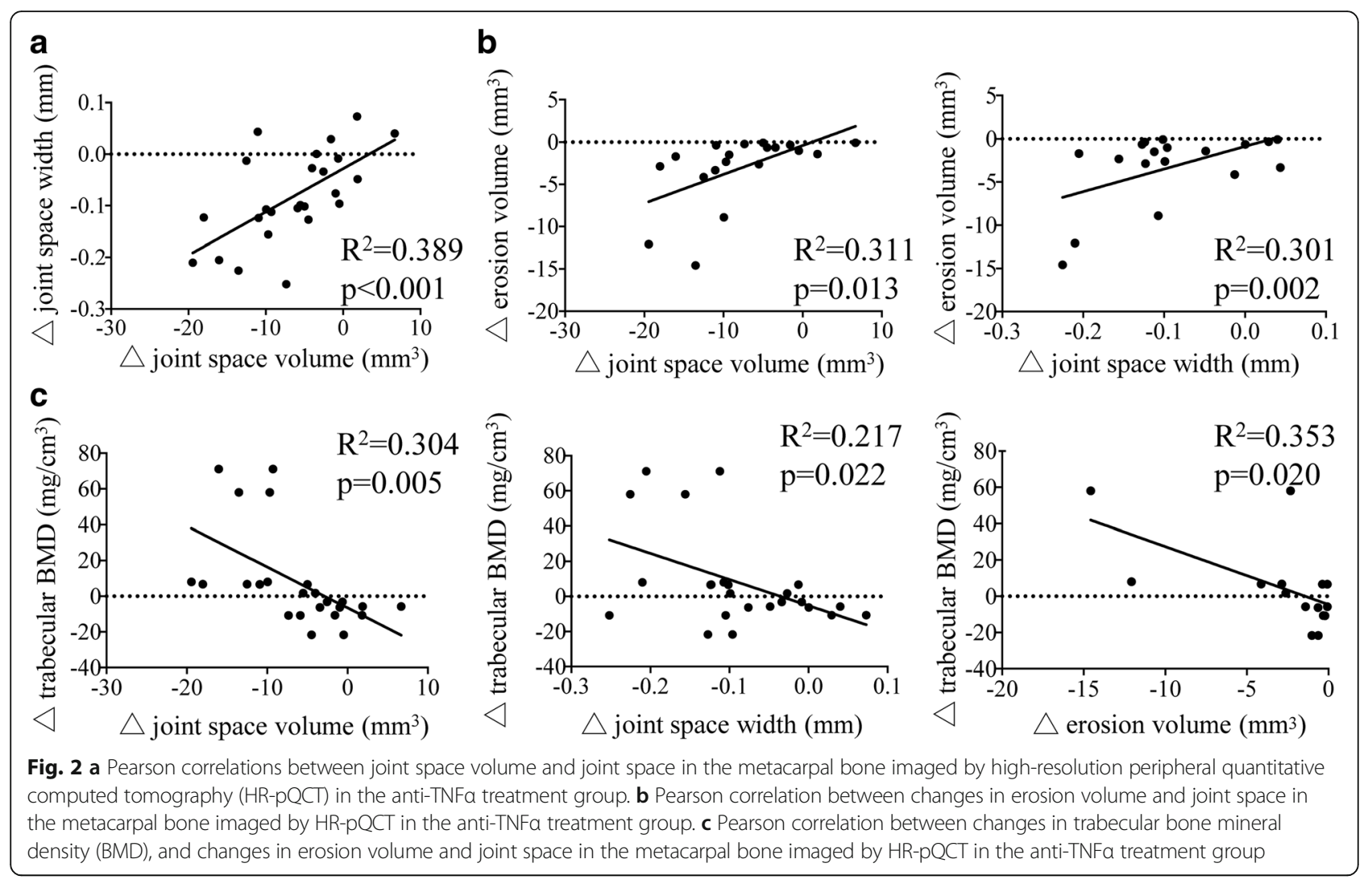

the wrist joint, none of the parameters were statistically significantly correlated (data not shown).

Next we investigated correlation between changes in bone erosion volume and clinical status. Because erosion volume and change varied between MCP and wrist joints, we calculated percentage changes in the total bone erosion of the MCP and wrist joint from baseline to 3 months and investigated correlation with the change in the DAS28 ESR and CRP (Fig. 3). In the anti-TNFo group, the percentage change in total erosion volume from baseline to 3 months was positively correlated, albeit statistically insignificant, with change in the DAS-28 ESR and CRP (Fig. 3a). In all patients, percentage change in total erosion volume from baseline to 3 months was statistically significantly and positively correlated with change in the DAS28 ESR and CRP (Fig. 3b).

\section{Radiography and MRI results}

Modified Sharp scores were $4.7 \pm 6.0$ in the MTX group vs. $26.1 \pm 42.0$ in the anti-TNF $\alpha$ group at baseline. The RAMRIS bone edema score decreased, albeit this was statistically insignificant, in the anti-TNF $\alpha$ group from baseline to 3 months. However, there were no differences in the other RAMRIS scores, including bone erosion score from baseline to 3 months, in either group (Table 2). No significant correlation was observed between change in HR-pQCT parameters and the RAMRIS scores.

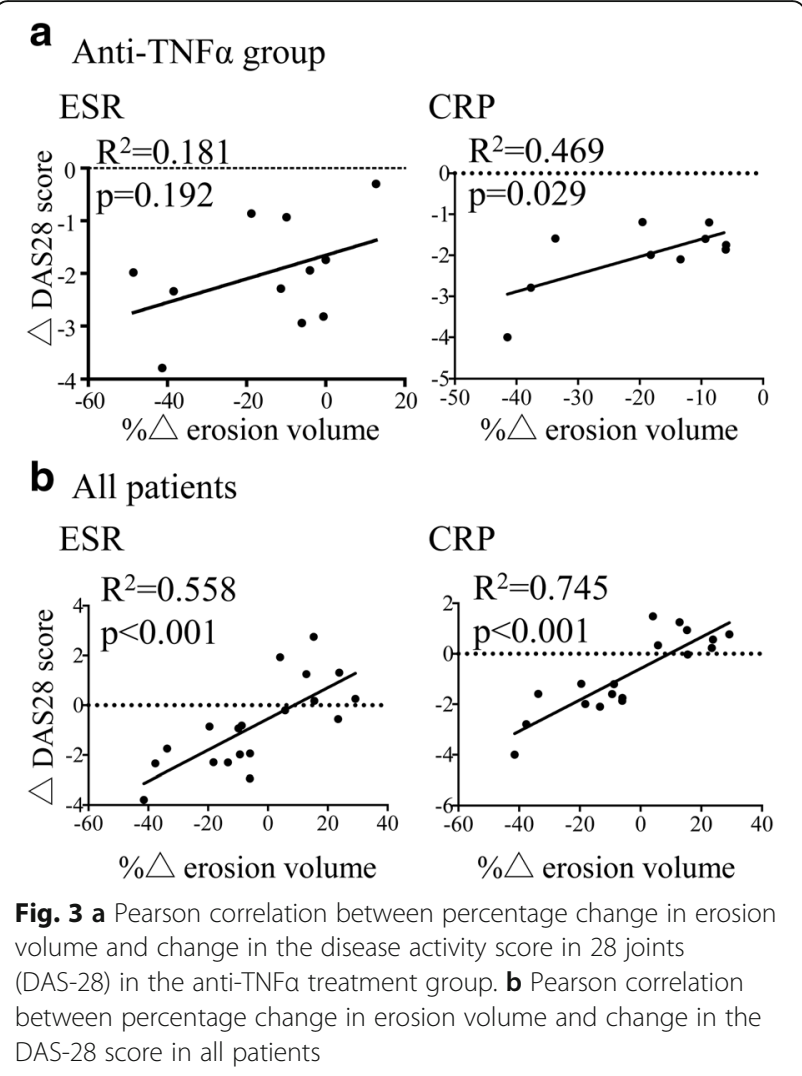




\section{Discussion}

To the best of our knowledge, this is the first HR-pQCT data characterizing and quantifying the early (3-month) effect of anti-TNF $\alpha$ treatment on bone erosion progression and bone microstructure in RA. In accordance with a significant decrease in the clinical DAS-28 scores in the anti-TNFa treatment group, erosion volumes and the joint space tended to decrease, especially in the MCP joints, suggesting that the bone changes are wellreflected in the clinical response. To date, there has only been a single retrospective HR-pQCT study reporting a significant reduction in erosion depth in patients with RA treated with anti-TNFo for one year [28]. Our data showed a tendency toward decreased erosion volume even though patients were on anti-TNF $\alpha$ treatment for only 3 months; in this regard, our results confirm and expand on the previous report [28]. Additionally, our findings are consistent with previous studies that showed anti-TNF $\alpha$ treatments prevent erosion progression compared to treatment with MTX alone [4-7], although we showed it as early as 3 months, while previous studies usually utilized 1-year-old to 2-year-old radiographic findings. Therefore, the high sensitivity of HR-pQCT could potentially help to reduce the length of future clinical trials.

Interestingly, even though the anti-TNF $\alpha$ group had higher disease activity than the MTX-only group at both baseline and 3 months, the anti-TNF $\alpha$ treatment seems to have prevented an increase in bone erosion in this group. This could for example be explained by the hypothesis that anti-TNF $\alpha$ might not only have suppressed inflammation but also osteclastogenesis, which is one of the major pathologic steps in RA pathogenesis [1], and the RANK-RANKL complex is a principal regulator and is activated in the early phase of osteoclast differentiation [29]. TNF $\alpha$ is a major proinflammatory cytokine in RA and is reported to influence osteoclast precursor cells through the expression or the activation of RANK [30]. Certainly, considered with the significant positive correlation between change in erosion volume and the DAS-28 scores, control of disease activity is thought to be important to prevent bone destruction, and this is supported by our observation of increased erosion volume in a patient who was not responding clinically to the anti-TNF treatment (Fig. 4). However, a recent randomized controlled trial showed that denosumab, a RANKL inhibitor, decreases bone erosion volume within 6 months in patients with moderately controlled RA, but not within 3 months [31]. Therefore, our findings suggest that anti-TNF $\alpha$ could have an even earlier effect in preventing and restoring arthritis-induced bone destruction if patients respond to the therapy.

The changes in erosion volume were correlated with the changes in joint space and trabecular BMD of the

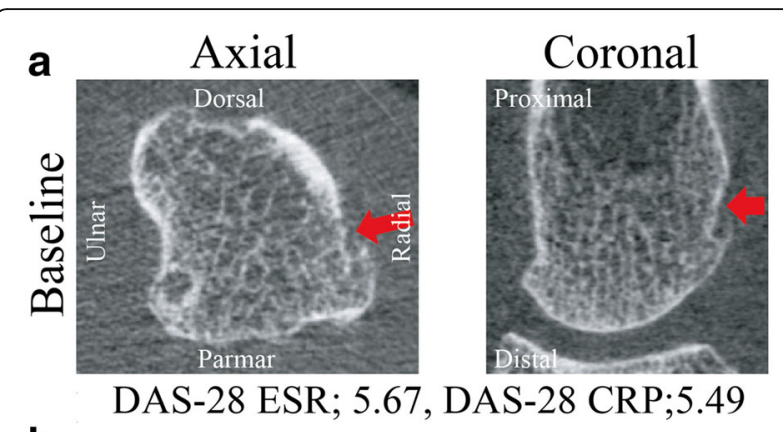

b

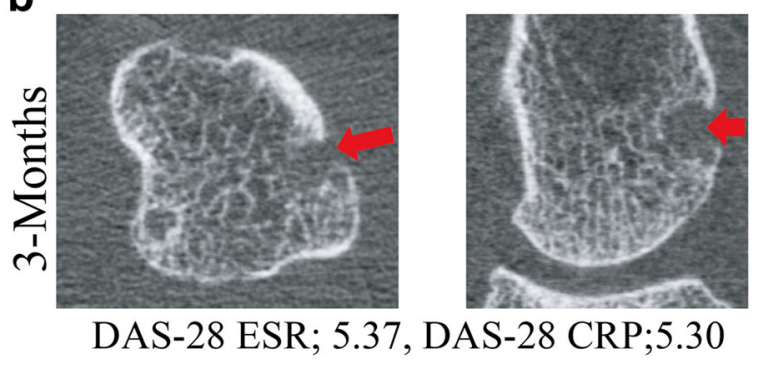

Fig. 4 Axial and coronal high-resolution peripheral quantitative computed tomography (HR-pQCT) images at the second metacarpal head in a patient who demonstrated increased erosion (erosion volume $=3.90 \mathrm{~mm}^{3}$ at baseline $(\mathbf{a})$ and $12.38 \mathrm{~mm}^{3}$ at 3 months $(\mathbf{b})$ ). Red arrows indicate bone erosion. DAS-28 disease activity score in 28 joints

MCP joints from baseline to 3 months in the anti-TNF $\alpha$ group. Although joint space narrowing is thought to be one of the typical radiographic characteristics in patients with RA [32], interestingly the anti-TNF $\alpha$ group had a significant decrease in joint space volume at the MCP joints following clinical response to therapy. It could be explained by the reduced amount of effusion and inflammation in the joint following anti-TNFo treatment in this cohort. However, the joint space width in the MTXonly group was smaller than in the anti-TNF group. The reason was thought to be that the anti-TNF group included some patients with subluxation, who had wider joint spaces. Therefore, the joint space changes may not be linear in RA, depending on the stage of disease and treatment. We also found that a decrease in erosion volume was correlated with an increase in trabecular BMD and structures, consistent with an overall bone damage prevention effect with anti-TNF treatment, as discussed previously.

Although we observed moderate to strong correlation between changes in erosions and changes in joint space and trabecular BMD in the MCP joints, we did not identify similar correlation for the wrist joints. However, the wrist joint is more complicated, including radio-lunate, radio-scaphoid and radio-ulnar joints, and is wider than the MCP joints, so it might be more difficult to capture rapid changes in bone destruction. Additionally, the wrist joints are much more dependent than the MCP 
joints on abduction and adduction effects, as the wrist joint allows joint movement in two planes, which are not possible in the hinged (ginglymus) MCP joints which can only be moved in one plane.

Of note, our data did not show any significant correlation between HR-pQCT erosion volume changes and changes in MR-based RAMRIS scores. This suggests that MRI and HR-pQCT are two distinct imaging modalities with different strengths and focus to evaluate RA pathophysiology. MRI measures, especially synovitis and BME have been suggested to be indicators of inflammation within the joints in RA. However, in terms of RAMRIS synovitis and BME scores, the range of scoring is too small $(0-3)$ to show significant correlation with the changes in HR-pQCT parameters in this study. Therefore, more sensitive quantitative MRI analysis such as synovitis volume and BME-like lesion volume might be necessary for comparison with these HR-pQCT-driven parameters. On the other hand, in terms of bone erosion, a recent study reported that MRI has lower sensitivity in the detection of bone erosions (60\%) and detection of osteosclerosis (24\%) compared to HRpQCT in the MCP2, MCP3 and wrists of patients with RA [33]. Additionally, a recent systemic review reported that RAMRIS erosions and joint-space narrowing scores could not detect early significant changes [34]. Therefore, focusing on bone-related changes, HR-pQCT is a powerful tool for providing objective and sensitive measures of bone erosions, cortical and trabecular bone structure and joint space morphology. These HR-pQCTderived imaging markers are promising outcome measures for future potential trials that combine treatment of inflammation and bone damage (anti-absorption treatment, for example) in RA.

There are some limitations of this study to report: first, HR-pQCT has a limited field of view (FOV), as compared to other imaging modalities such as MRI, whole body CT or cone-beam radiographic imaging systems, which are also more widely available than HRpQCT. Furthermore, HR-pQCT is normally limited to a small number of joints, primarily due to keeping the overall scan within a clinically feasible time. In future studies, mid-carpal joint and more distal regions may be considered as well. Second, this study was limited by its small sample size, but this is the first report about applying HR-pQCT at 3 months after anti-TNFo treatment and was meant to be exploratory in nature. In the future, larger cohort studies are warranted to confirm the findings from this study. Third, the study was also limited by a follow up of 3 months. Although we targeted the early changes in bone during treatment with anti-TNF $\alpha$ in this study, we are currently following up these patients at 1 year after treatment to investigate long-term bone changes using HR-pQCT. Finally, bone turnover markers were not checked in this study. Bone markers e.g. tartrate-resistant acid phosphatase 5b (TRAP5b) have been shown to correlate with bone erosions in patients with RA imaged by HR-pQCT [35]. Although there are some risk factors that influence bone turnover markers in patients with RA, such as steroid use and immobility, it should be addressed in future studies as to whether bone markers are useful in predicting changes in erosion volume in patients with RA who are receiving anti-TNF $\alpha$.

\section{Conclusions}

Using HR-pQCT we demonstrated that anti-TNF $\alpha$ treatment can prevent an increase in bone erosion within a 3-month treatment period, consistent with a significant decrease in disease activity. Our results suggest that HRpQCT is a sensitive and powerful tool for quantifying bone changes and monitoring RA treatment even within a short-term time window such as 3 months. However, future larger HR-pQCT studies with follow-up periods of 3 months or less are needed to validate these initial observations.

\begin{abstract}
Abbreviations
BMD: Bone mineral density; BME: Bone marrow edema; CRP: C-reactive protein; CT: Computed tomography; CV: Coefficient variance; DAS: Disease activity score; DICOM: Digital imaging and communications in medicine; ESR: Erythrocyte sedimentation rate; FSE: Fast spin echo; HR-pQCT: Highresolution peripheral quantitative computed tomography; IDEAL: Iterative decomposition of water and fat with echo asymmetry and least-squares estimation; IL: Interleukin; JSW: Joint space width; LSC: Least significant change; MCH: Distal head of the metacarpal; MCP: Metacarpophalangeal; MRI: Magnetic resonance imaging; MTX: Methotrexate; PB: Phalangeal base; RA: Rheumatoid arthritis; RAMRIS: OMERACT Outcome Measures in Rheumatology (OMERACT) rheumatoid arthritis-magnetic resonance imaging scoring; SPGR: Spoiled gradient echo; TE: Echo time; TNFa: Tumor necrosis factor alpha; TR: Repetition time
\end{abstract}

Acknowledgements

We thank all patients who participated in this study.

Funding

The study was supported by UCB Pharma Inc.

Availability of data and materials

The datasets used and/or analyzed during the present study are available from the corresponding author on reasonable request.

\section{Authors' contributions}

TS and HJC equally contributed to reading HR-pQCT images, performed the analysis, interpreted the results and wrote the manuscript. UH performed image analysis, manuscript drafting and review, figure creation, data access, data collection, data interpretation and submission. MT contributed to the analysis of bone parameters and technical coordination. AJB contributed to data collection, interpretation of the results and technical coordination. JSG contributed to reading HR-pQCT images for inter-reader reproducibility. NC and TML contributed to reading the radiographs and MRI scans. JG and JBM contributed to the design of the study, recruitment of patients and clinical assessment. XL contributed to the design of the study and interpretation of the results and securing funding. Approval of the final version of the manuscript was obtained from all co-authors. 


\section{Ethics approval and consent to participate}

The Institutional Review Board for Human Research approved this study design (IRB\# 12-10418) and written informed consent was obtained from all subjects prior to participate in study.

\section{Consent for publication}

Not applicable.

\section{Competing interests}

The authors declare that they have no competing interests.

\section{Publisher's note}

Springer Nature remains neutral with regard to jurisdictional claims in published maps and institutional affiliations.

\section{Author details}

${ }^{1}$ Department of Radiology \& Biomedical Imaging, Musculoskeletal Quantitative Imaging Research, University of California San Francisco, 185 Berry Street, Suite 350, San Francisco, CA 94107, USA. ${ }^{2}$ Department of Orthopedic Surgery, Graduate School of Medicine, Hokkaido University, Sapporo, Japan. ${ }^{3}$ Department of Internal Medicine, Division of Rheumatology, Gachon University Gil Hospital, Incheon, Korea. ${ }^{4}$ Department of Medicine, Division of Rheumatology, University of California San Francisco, San Francisco, California, USA.

Received: 18 May 2017 Accepted: 15 September 2017 Published online: 04 October 2017

\section{References}

1. Mclnnes IB, Schett $G$. The pathogenesis of rheumatoid arthritis. N Engl J Med. 2011;365(23):2205-19.

2. Machold KP, Stamm TA, Nell VP, Pflugbeil S, Aletaha D, Steiner G, Uffmann $M$, Smolen JS. Very recent onset rheumatoid arthritis: clinical and serological patient characteristics associated with radiographic progression over the first years of disease. Rheumatology (Oxford). 2007:46(2):342-9.

3. Schett G, Gravallese E. Bone erosion in rheumatoid arthritis: mechanisms, diagnosis and treatment. Nat Rev Rheumatol. 2012;8(11):656-64.

4. Lipsky PE, van der Heijde DM, St Clair EW, Furst DE, Breedveld FC, Kalden JR, Smolen JS, Weisman M, Emery P, Feldmann M, et al. Infliximab and methotrexate in the treatment of rheumatoid arthritis. Anti-Tumor Necrosis Factor Trial in Rheumatoid Arthritis with Concomitant Therapy Study Group. N Engl J Med. 2000;343(22):1594-602.

5. Keystone EC, Kavanaugh AF, Sharp JT, Tannenbaum H, Hua Y, Teoh LS, Fischkoff SA, Chartash EK. Radiographic, clinical, and functional outcomes of treatment with adalimumab (a human anti-tumor necrosis factor monoclonal antibody) in patients with active rheumatoid arthritis receiving concomitant methotrexate therapy: a randomized, placebo-controlled, 52week trial. Arthritis Rheum. 2004;50(5):1400-11.

6. Klareskog L, van der Heijde D, de Jager JP, Gough A, Kalden J, Malaise M, Martin Mola E, Pavelka K, Sany J, Settas L, et al. Therapeutic effect of the combination of etanercept and methotrexate compared with each treatment alone in patients with rheumatoid arthritis: double-blind randomised controlled trial. Lancet. 2004;363(9410):675-81.

7. Emery P, Fleischmann R, van der Heijde D, Keystone EC, Genovese MC, Conaghan PG, Hsia EC, Xu W, Baratelle A, Beutler A, et al. The effects of golimumab on radiographic progression in rheumatoid arthritis: results of randomized controlled studies of golimumab before methotrexate therapy and golimumab after methotrexate therapy. Arthritis Rheum. 2011;63(5): 1200-10.

8. Baker JF, Ostergaard M, Emery P, Hsia EC, Lu J, Baker DG, Conaghan PG. Early MRI measures independently predict 1-year and 2-year radiographic progression in rheumatoid arthritis: secondary analysis from a large clinical trial. Ann Rheum Dis. 2014;73(11):1968-74.

9. Hirota T, Suzuki T, Ogishima H, Hagiwara S, Ebe H, Takahashi H, Yokosawa $\mathrm{M}$, Umeda $\mathrm{N}$, Kondo $\mathrm{Y}$, Tsuboi $\mathrm{H}$, et al. Evaluation of changes in magnetic resonance images following 24 and 52 weeks of treatment of rheumatoid arthritis with infliximab, tocilizumab, or abatacept. Mod Rheumatol. 2016; 26(1):29-35.

10. Barnabe C, Finzel S, Stok KS, Geusens P. High-resolution peripheral quantitative CT in rheumatology. Nat Rev Rheumatol. 2015;11(2):123.
11. Kleyer A, Finzel S, Rech J, Manger B, Krieter M, Faustini F, Araujo E, Hueber AJ, Harre U, Engelke $K$, et al. Bone loss before the clinical onset of rheumatoid arthritis in subjects with anticitrullinated protein antibodies. Ann Rheum Dis. 2014;73(5):854-60.

12. Finzel S, Englbrecht M, Engelke K, Stach C, Schett G. A comparative study of periarticular bone lesions in rheumatoid arthritis and psoriatic arthritis. Ann Rheum Dis. 2011;70(1):122-7.

13. Finzel S, Sahinbegovic E, Kocijan R, Engelke K, Englbrecht M, Schett G. Inflammatory bone spur formation in psoriatic arthritis is different from bone spur formation in hand osteoarthritis. Arthritis Rheum. 2014, 66(11):2968-75.

14. Moller Dohn U, Boonen A, Hetland ML, Hansen MS, Knudsen LS, Hansen A, Madsen OR, Hasselquist M, Moller JM, Ostergaard M. Erosive progression is minimal, but erosion healing rare, in patients with rheumatoid arthritis treated with adalimumab. A 1 year investigator-initiated follow-up study using high-resolution computed tomography as the primary outcome measure. Ann Rheum Dis. 2009;68(10):1585-90.

15. Aletaha D, Neogi T, Silman AJ, Funovits J, Felson DT. Bingham 3rd CO, Birnbaum NS, Burmester GR, Bykerk VP, Cohen MD, et al. 2010 Rheumatoid arthritis classification criteria: an American College of Rheumatology/ European League Against Rheumatism collaborative initiative. Arthritis Rheum. 2010;62(9):2569-81.

16. Wells G, Becker JC, Teng J, Dougados M, Schiff M, Smolen J, Aletaha D, van Riel PL. Validation of the 28-joint Disease Activity Score (DAS28) and European League Against Rheumatism response criteria based on C-reactive protein against disease progression in patients with rheumatoid arthritis, and comparison with the DAS28 based on erythrocyte sedimentation rate. Ann Rheum Dis. 2009;68(6):954-60.

17. Srikhum W, Virayavanich W, Burghardt AJ, Yu A, Link TM, Imboden JB, Li X. Quantitative and semiquantitative bone erosion assessment on highresolution peripheral quantitative computed tomography in rheumatoid arthritis. J Rheumatol. 2013;40(4):408-16.

18. Boutroy S, Bouxsein ML, Munoz F, Delmas PD. In vivo assessment of trabecular bone microarchitecture by high-resolution peripheral quantitative computed tomography. J Clin Endocrinol Metab. 2005;90(12):6508-15.

19. Pialat JB, Burghardt AJ, Sode M, Link TM, Majumdar S. Visual grading of motion induced image degradation in high resolution peripheral computed tomography: impact of image quality on measures of bone density and micro-architecture. Bone. 2012;50(1):111-8.

20. Laib A, Hauselmann HJ, Ruegsegger P. In vivo high resolution 3D-QCT of the human forearm. Technol Health Care. 1998;6(5-6):329-37.

21. Yang H, Yu A, Burghardt AJ, Virayavanich W, Link TM, Imboden JB, Li X. Quantitative characterization of metacarpal and radial bone in rheumatoid arthritis using high resolution-peripheral quantitative computed tomography. Int J Rheum Dis. 2017;20(3):353-362.

22. Burghardt AJ, Lee CH, Kuo D, Majumdar S, Imboden JB, Link TM, Li X. Quantitative in vivo HR-pQCT imaging of 3D wrist and metacarpophalangeal joint space width in rheumatoid arthritis. Ann Biomed Eng. 2013;41(12):2553-64.

23. Barnabe C, Feehan L. High-resolution peripheral quantitative computed tomography imaging protocol for metacarpophalangeal joints in inflammatory arthritis: the SPECTRA collaboration. J Rheumatol. 2012;39(7): 1494-5.

24. van der Heijde DM, van Riel PL, Nuver-Zwart IH, Gribnau FW, vad de Putte LB. Effects of hydroxychloroquine and sulphasalazine on progression of joint damage in rheumatoid arthritis. Lancet. 1989;1(8646):1036-8.

25. Ory PA. Interpreting radiographic data in rheumatoid arthritis. Ann Rheum Dis. 2003:62(7):597-604.

26. Conaghan P, Bird P, Ejbjerg B, O'Connor P, Peterfy C, McQueen F, Lassere M, Emery P, Shnier R, Edmonds J, et al. The EULAR-OMERACT rheumatoid arthritis MRI reference image atlas: the metacarpophalangeal joints. Ann Rheum Dis. 2005;64 Suppl 1:i11-21.

27. Ostergaard M, Edmonds J, McQueen F, Peterfy C, Lassere M, Ejbjerg B, Bird P, Emery P, Genant H, Conaghan P. An introduction to the EULAR-OMERACT rheumatoid arthritis MRI reference image atlas. Ann Rheum Dis. 2005;64 Suppl 1:i3-7.

28. Finzel S, Rech J, Schmidt S, Engelke K, Englbrecht M, Stach C, Schett G. Repair of bone erosions in rheumatoid arthritis treated with tumour necrosis factor inhibitors is based on bone apposition at the base of the erosion. Ann Rheum Dis. 2011;70(9):1587-93.

29. Boyle WJ, Simonet WS, Lacey DL. Osteoclast differentiation and activation. Nature. 2003;423(6937):337-42. 
30. Karmakar S, Kay J, Gravallese EM. Bone damage in rheumatoid arthritis: mechanistic insights and approaches to prevention. Rheum Dis Clin North Am. 2010;36(2):385-404.

31. Yue J, Griffith JF, Xiao F, Shi L, Wang D, Shen J, Wong P, Li EK, Li M, Li TK, et al. Repair of bone erosion in rheumatoid arthritis by denosumab: a highresolution peripheral quantitative computed tomography study. Arthritis Care Res. 2017;69(8):1156-1163.

32. van der Heijde D. Erosions versus joint space narrowing in rheumatoid arthritis: what do we know? Ann Rheum Dis. 2011;70 Suppl 1:11 16-8.

33. Regensburger A, Rech J, Englbrecht M, Finzel S, Kraus S, Hecht K, Kleyer A, Haschka J, Hueber AJ, Cavallaro A, et al. A comparative analysis of magnetic resonance imaging and high-resolution peripheral quantitative computed tomography of the hand for the detection of erosion repair in rheumatoid arthritis. Rheumatology (Oxford). 2015;54(9):1573-81.

34. Woodworth TG, Morgacheva O, Pimienta OL, Troum OM, Ranganath VK, Furst DE. Examining the validity of the rheumatoid arthritis magnetic resonance imaging score according to the OMERACT filter-a systemic literature review. Rheumatology. 2017;56(7):1177-88.

35. Aschenberg S, Finzel S, Schmidt S, Kraus S, Engelke K, Englbrecht M, Rech J, Schett G. Catabolic and anabolic periarticular bone changes in patients with rheumatoid arthritis: a computed tomography study on the role of age, disease duration and bone markers. Arthritis Res Ther. 2013;15(3):R62.

\section{Submit your next manuscript to BioMed Central} and we will help you at every step:

- We accept pre-submission inquiries

- Our selector tool helps you to find the most relevant journal

- We provide round the clock customer support

- Convenient online submission

- Thorough peer review

- Inclusion in PubMed and all major indexing services

- Maximum visibility for your research

Submit your manuscript at www.biomedcentral.com/submit 